\title{
Study on Automatic Switching Protection of Optical with 3R Amplier Based on FPGA
}

\author{
Xiong Li \\ Department of Network Engineering, Shanghai Qianqiao University, Shanghai, China
}

\section{Email address:}

17032@gench.edu.cn

\section{To cite this article:}

Xiong Li. Study on Automatic Switching Protection of Optical with 3R Amplier Based on FPGA. Science Discovery.

Vol. 6, No. 3, 2018, pp. 189-192. doi: 10.11648/j.sd.20180603.18

Received: May 5, 2018; Accepted: June 20, 2018; Published: June 26, 2018

\begin{abstract}
In this paper, we research and develop a new type of optical fiber based on high-speed switching FPGA routing automatic protection switching device, its innovative USES high-speed electronic SWITCH high-speed exchange of FPGA design, will be 0 to $10 \mathrm{~g}$ digital optical signal photoelectric conversion amplification and rearrangement of the equipment selected by the high speed switching FPGA SWITCH protection, can for any important 0 to $10 \mathrm{~g}$ optical signal circuit after transformation $(1+1)$ send after protect rearrangement of amplification, adopt concurrent optimization of transmission mode, automatic selection of high quality circuit signal in the microsecond time complete electrical SWITCH (far superior to the traditional common optical SWITCH SWITCH time), based on the development of commercial products for the national grid ehv transmission network users with high reliability,high performance of 0 to $10 \mathrm{~g}$ optical signal transmission channel protection, its there is no insertion loss, does not affect the original light path, also used in long-distance optical network transmission protection system in the west and subvert the traditional light path switching apparatus for at least $2 \sim 5$ DBM large insertion loss. It overcomes the disadvantage of the traditional optical switch protection device.
\end{abstract}

Keywords: High-Speed Exchange, FPGA, OPTICAL SWITCH, 3R Regeneration, Digital Signal OPTICAL Path Switching, Insertion Loss

\section{基于自主FPGA芯片的光纤路由无损 3R 自动切换的设计}

\section{李雄}

信息学院网络工程系, 上海建桥学院, 上海, 中国

\section{邮箱}

17032@gench.edu.cn

摘要: 本文主要研究和研制一种新型基于自主FPGA FPGA-FPGA (Field-Programmable Gate Array),即现场可编程门阵列 芯片的光纤路由无损 $3 R$ 自动切换装置, 其创新地采用电子高速开关高速交换 CPLD设计, 将0-10G数字光信号光电转 换放大并由高速交换FPGA保护选择倒换设备, 可以对于任意重要的 $0-10 G$ 光信号电路转换后进行 (1+1) 放大发送后 保护倒换, 采取并发优收的传输方式, 自动选择质量高的线路信号, 在微秒级的时间内完成电切换（远优于传统普通 的光开关切换时间），基于此研制的商用产品为国家电网超高压传输网用户提供高可靠、高性能的0-10G光信号保护 传输通路, 其不存在插损, 不影响原光路, 也用于西部长距离光纤网传输保护系统中, 颠覆了传统光路切换仪至少 2 5dbm 的较大插入损耗。摆脱了对国外专用芯片的限制, 克服了传统的光开关保护仪的插损大、倒换时间长的缺点。

关键词：高速交换FPGA, CPLD, OPTICAL SWITCH, 3R再生, 数字信号, 光路切换, 插损 


\section{1. 引言}

在信息时代, 现代化大容量通信光缆网已连接到千 家万户。但是在竞争日趋激烈的电信市场中, 仅有大容 量通信能力是远远不够的, 更重要的是服务质量与网络 性能等核心方面的竞争,好的网络性能可为每一个客户的 通信畅通无阻提供保障。所以应采取什么样的技术措施 和方案来保证大数据的光纤光缆通信无阻断, 特别是传 输干线的重要通信, 从而保护用户的根本利益, 是我们面 临的重要课题。

在已建成的长距离大容量的主干高速光纤通信网上 实现安全通信, 除传输设备因素外, 首先还要考虑的就 是对光传输物理路由实施自动保护的技术的解决方案,

其次方案应具有安全可靠、保护迅速、具有强大的抗灾 害和抗阻断能力, 并且需要投资少、见效大, 在网络安 全凸显越来越重要的今天, 具有高度应用推广价值。目 前光路保护仪均常采用的是 1 X2、2X2机械光开关器件, 但采用传统的光开关的保护仪具有的插损大，倒换时间 长的缺点。其不适应光强在临界点附近的场景。

\section{2. 保护技术介绍及研究现状}

(1)光路SDH系统的自愈保护技术

SDH经典的保护倒换已得到普遍认同。通过SDH自 愈环的组网结构, 环上的各个节点能够根据业务量的需 要灵活地上下电路; 同时电路可 $100 \%$ 的得到保护, 无需 人为干预, 网络便能从失效的故障中实时地自动恢复业 务, 从而真正实现了自愈功能。

然而与信号和软件密切相关, 且需要成环的四芯两 两保护, 成本高, 且需要多端和网上均采用同一厂家的 设备, 局限行非常大。

(2)人工调度保护

所谓人工调度保护, 就是在光缆干线发生障碍后, 根据光缆应急预案, 通过机务与线务部门的配合,采用同 方向其他光缆线路迂回调度，人工方式抢通受阻光缆干 线的业务使用系统。人工调度保护需要机务部门的大力 支持和积极配合, 而且手工倒通电路不仅要求要有值班 人员在场, 同时值班人员要具备一定的电路抢修意识、 业务水平和动手操作能力。根据目前的维护体制, 地市 级部门有专业的维护人员, 可达到上述要求; 但对于无 人职守站和县镇级的中继站机房, 由于成为综合值班维 护, 人员技术水平与实战能力均不如地市站的维护人员, 因此在故障发生时无法满足人工快速倒通电路的要求, 业务恢复时间较长。

\section{(3)光路自动切换保护技术}

光路自动切换保护技术是通过对光缆中传输光功率 变化的实时监视、告警信息的自动分析, 能够及时发现 故障及隐患, 在出现严重故障时, 快速将工作光路自动 切换到备用通道, 在极短的时间内恢复通信, 完成对光 缆故障的快速反应和恢复机制。

该保护技术有两种基本实现方式:
1) 目前常采用的是 $1 X 2 、 2 X 2$ 机械光开关器件, 但 采用传统的光开关的保护仪具有的插损大, 倒换时间长 的缺点。研究现状调研市场上均采用这种模式, 其使用 的场景非常局限。

2) 采用高速交换FPGA SWITCH高速交换开关, 由 于电子级比机械级倒换速度快, 且有放大再生功能, 所 以本文特别讨论采用这种交换开关技术的优点和原理。

\section{3. 本设计的实现原理与实施方式}

本研究的如图1的结构设计, 这种具有信号收发功能 的高速电子开关型光缆保护仪, 是应用于同样具有收发 和传递信号功能的光缆终端设备、主用和备用光缆终端 设备之间, 并通过人机接口与外围 $\mathrm{PC}$ 机进行信号传递与 控制, 其组成 (如图1) 包括: 第一光转电单元, 第二光 转电单元, 高速信号交叉切换单元, 3R 放大单元, 设备 监控单元, 电转光单元和电源单元;

第一光转电单元接收主用光缆所发送的主用光缆光 信号并转换为主用电信号发送给高速信号交叉切换单元, 同时发送光转电主用状态信号给设备监控单元; 同样, 第二光转电单元接收备用光缆所发送的备用光缆光信号 并转换为备用电信号发送给高速信号交叉切换单元，同 时发送光转电备用状态信号给设备监控单元;

两个光转电单元是为了便于硬件电路对信号的处理, 其组成包括光学次模块 (Optical Subassembly; OSA) 及 电子次模块 (Electrical Subassembly; ESA) 两大部分; 首先光学次模块部分是以砷化镓 (GaAs) 、磷化铟 (InP) 、砷化铟镓 (InGaAs) 等作为发光与检光材料, 形成光信号; 电子次模块内部包含传送驱动 IC及接收驱 动IC, 分别用以驱动雷射二极管与检光二级管, 形成电 信号; 从而使得主用备用光缆来的光信号可以转换为电 信号。

设备监控单元是由网管板、CPLD和MPU组成, MPU接收所述光转电主用状态信号和光转电备用状态信 号进行处理获得切换控制信号并由CPLD发送给高速信号 交叉切换单元; 若设备监控单元本身没有自动发送切换 控制信号给高速信号交叉切换单元, 则外围PC机根据光 转电主用状态信号和光转电备用状态信号主动发送切换 控制指令给高速信号交叉切换单元;

高速信号交叉切换单元接收主用电信号、备用电信 号和切换控制信号, 并根据切换控制信号选择主用电信 号和备用电信号中的一路电信号作为光缆终端设备所需 电信号发送给3R放大单元;

高速信号交叉切换单元是由比较器、微处理器和电 子开关组成, 微处理器根据切换控制信号对主用电信号 和备用电信号进行质量判断与比较, 若主用电信号和备 用电信号都存在, 则通过比较器选择主用电信号和备用 电信号中电流值较大的一路电信号作为光缆终端设备所 需电信号传递给电子开关进行传输; 电子开关是由两个 三极管门电路组成, 且电子开关的控制电极是由微处理 器控制 0 或 1 输出控制通断; 
若主用电信号和备用电信号中有一路电信号丢失, 则将另一路电信号作为光缆终端设备所需电信号传递给 电子开关进行传输;

若光主用电信号和备用电信号都丢失, 则电子开关 无动作。

$3 R$ 放大单元接收光缆终端设备所需电信号进行重定 时、重再生和重整形处理, 获得3R电信号传递给电转光 单元; $3 R$ 放大单元采用FPGA作为主处理芯片, 为设备设 计提供离散速率、多种速率和连续可调的时钟和数据恢 复集成电路, 能够轻松集成协议未知的应用, 并自动锁 定速率在 $12.3 \mathrm{Mbps} \sim 2.7 \mathrm{Gbps}$ 的输入光转电数据流, 无需 参考时钟, 同时通过串行接口报告获得的光电信号数据 速率。

电转光单元获得 $3 R$ 电信号进行电光转换后获得 $3 R$ 光 信号传递给光缆终端设备光信号的收光端; 同时将电转 光状态信号发送给设备监控单元; 电转光状态信号包括 光有无信号, 光电流强度, 光功率强度, 光速率信号。

无源分光单元采用一分二的无源分光器接收光缆终 端设备发光端的光信号进行 $50 \%$ 的两路分光处理, 获得 主用发送光信号和备用发送光信号分别发送给主用光缆 和备用光缆用于主用和备用光缆终端设备之间进行传输; 从而实现对电转光单元输出来的 $3 \mathrm{R}$ 光信号的分两路后进 入光缆。
设备监控单元的MPU将所接收的电转光信号状态、 光转电主用电信号和光转电备用状态信号传递给所述网 管板进行转换, 转换为SNMP网管信息并经过所述人机接 口发送给所述外围 $\mathrm{PC}$ 机进行图形显示; 方便实时了解网 络状况和保护仪状况。

电源单元用于为第一光转电单元、第二光转电单元、 高速信号交叉切换单元、3R放大单元、设备监控单元和 电转光单元提供系统电源。系统电源是指接入交流 $220 \mathrm{~V}$ 或/和直流-48V, 然后输出各单元所需要的电源; 保证保 护仪得到交流220V和直流-48电源的同时备份供电, 确保 保护仪的稳定可靠运行。

保护仪引入了一分二的无源分光器单元, 实现了设 备自身放大后的的光信号进行复制和发送。

具体组网实施中, 如果需要多路切换, 可以采用单 元化的系统结构, 使第一第二光电转换单元、电光转换 单元、信号3R放大单元、信号高速交叉切换单元、无源 分光路单元、设备监控单元和电源单元及外部显示设备 成了一个有机的整体。在两端的两台光端设备之间插入 两台高速电子开关光保护仪, 在两台高速电子开关保护 仪之间接入一对保护用备用光缆, 即可以实现在主用光 缆和备用光缆之间进行无损自动瞬时切换。并远程实时 监控设备的各项状态, 切实保护电力通信设备稳定运行。

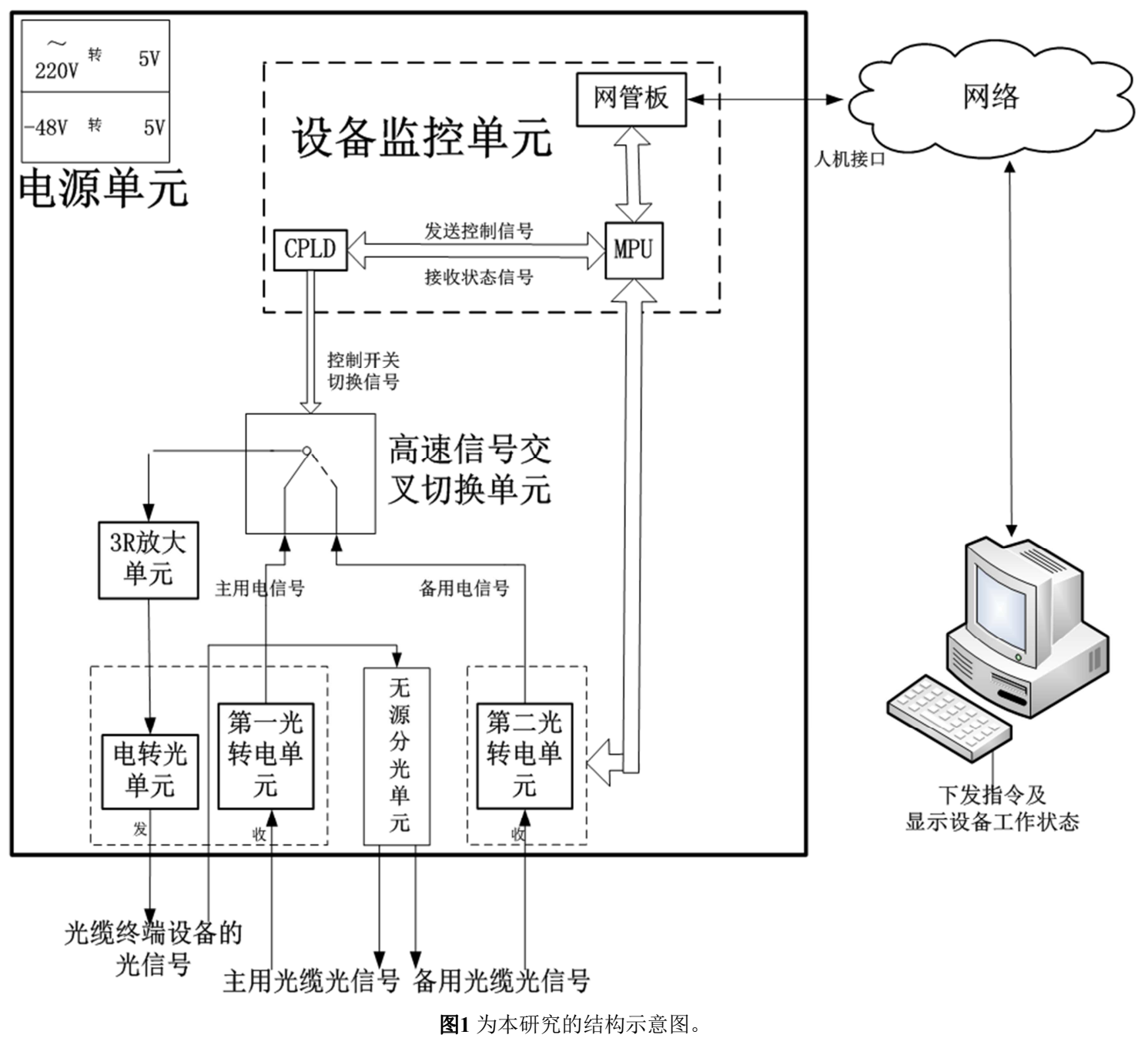


具体组网实施中, 如果需要多路切换, 可以采用单 元化的系统结构, 使第一第二光电转换单元、电光转换 单元、信号3R放大单元、信号高速交叉切换单元、无源 分光路单元、设备监控单元和电源单元及外部显示设备 成了一个有机的整体。在两端的两台光端设备之间插入 两台高速电子开关光保护仪, 在两台高速电子开关保护 仪之间接入一对保护用备用光缆, 即可以实现在主用光 缆和备用光缆之间进行无损自动瞬时切换。并远程实时 监控设备的各项状态, 切实保护电力通信设备稳定运行。

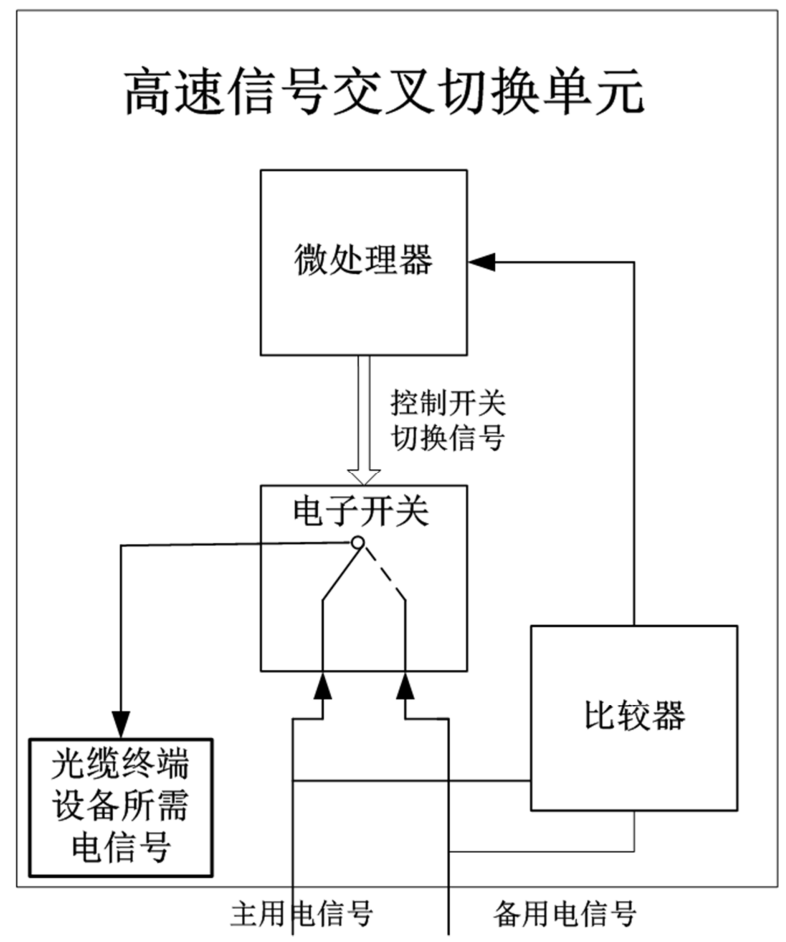

图2 为本研究中高速信号交叉切换单元的结构设计示意图. 详见作者专 利参考文献 9 。

\section{4. 研究设计总结与应用}

本方案和研究出来的设备已成功应用于国家电网特 高压电网、部分省级电力、电信等专线业务或其它重要 的光传输业务中。本方案已获得2013年发明专利, 本光 切换的使用提高了重要光网络的安全性, 在技术上要满 足了备用光路由通道、光切率在接入时的衰耗弥补、光 通道网管的监控通道等。

\section{五、结论}

基于FPGA自主设计的光路保护系统是针对光路线路 故障设计的, 是完全独立于SDH系统和DWDM系统的网 元设备之外的。在结合备用光纤路由或空闲波长通道的情 况下, 可以组建切换保护网路。通过实践证明光自动切换 保护具有快速可靠、安全灵活、业务恢复能力强的优点。 同时在切换保护网管与SDH设备网管的配合下, 为干线光 网络无阻断通信提供了实用、安全、经济的解决方案。
未来针对不断升级的电信、电力等光传输网络中, 我们将进一步提供更大型更高速率的方案和设备, 在光 缆条件复杂, 设备型号众多的光纤传输网中, 基于高速 FPGA芯片的光纤自动切换保护领域拥有比SDH环保护、 光开关型保护更大的使用前景；进而保证使网络更快、 更好、更安全。所以, 基于高速FPGA 3R SWITCH芯片 的光纤自动切换保护的前景更为看好。

\section{参考文献}

[1] 谷昕;利用光纤通信网络传送继电保护信号 [J]; 电力系统通 信;2014年07期。

[2] 吴湛;光路自动切换保护技术的原理和应用设想 [J];电力系 统通信;2016年02期。

[3] 丁慧霞;陈希; 光纤通道传输继电保护信号误码性能研究 [J]; 电力系统通信;2008年02期。

[4] 朱涁浅谈光纤通信系统监测 [J];电子商务;2011年12期。

[5] 李志东;ECM-FOMS光缆自动监测和管理系统原理 [J];广东 输电与变电技术; 2007 年 04 期。

[6] 王尔;光传输网络保护倒换[J];硅谷;2012年06期。

[7] 江明珠;徐庆富;李长俊;1:1方式带备纤监控的光路检测系统 研制[J];光通信技术;2011年01期。

[8] 全光3R再生系统理论分析和实验研究2017王耀天-《天津 大学学报》。

[9] 具有 $3 r$ 放大功能的光保护切换单元 2013 年专利作者张引强, 卓文合, 潘子春, 陈是同, 李雄。

[10] 用于光线路保护系统的带osc放大的edfa装置安运志2015。

[11] An all-optical 3R regenerator for 40-Gbit/s system based onsignal-pump fiber optical parametric amplification 基于信 号抽运的光纤光参量放大的全光3R再生系统 Wang $\mathrm{Ju}$ 物 理学报2017。

[12] 一种具有光放大功能的滤波器设计梁瑞生半导体光电2016。

\section{作者简介}

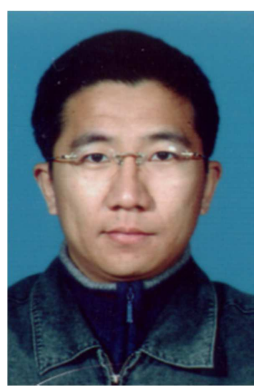

李雄, 男, 1973年生, 中国科学院上 海技物所物理电子学博士毕业, 从事 国企外企科研与设计二十余年, 现任 教于上海建桥学院网络工程系; 研究 方向是物联网、高速光网络、光交 换、光定位、嵌入式智能网关等。 